CrossMark \& click for updates

Cite this: Mol. BioSyst., 2015, 11,1612

Received 19th December 2014, Accepted 12th March 2015

DOI: $10.1039 / \mathrm{c} 4 \mathrm{mb} 00725 \mathrm{e}$

www.rsc.org/molecularbiosystems

\section{Proteomic analysis of human glioblastoma cell lines differently resistant to a nitric oxide releasing agent $\dagger$}

\author{
Roberta Leone, ${ }^{a}$ Paola Giussani, ${ }^{\mathrm{b}}$ Sara De Palma, ${ }^{\mathrm{c}}$ Chiara Fania, ${ }^{\mathrm{a}}$

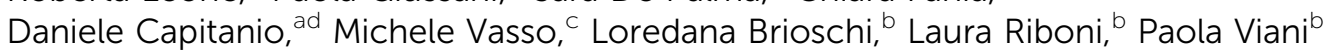 \\ and Cecilia Gelfi*acd
}

\begin{abstract}
Glioblastoma multiforme is the most aggressive astrocytoma characterized by the development of resistant cells to various cytotoxic stimuli. Nitric oxide (NO) is able to overcome tumor resistance in PTEN mutated rat C6 glioma cells due to its ability to inhibit cell growth by influencing the intracellular distribution of ceramide. The aim of this study is to monitor the effects of NO donor PAPANONOate on ceramide trafficking in human glioma cell lines, CCF-STTG1 (PTEN-mutated, p53-wt) and T98G (PTENharboring, p53-mutated), together with the assessment of their differential molecular signature by 2D-DIGE and MALDI mass spectrometry. In the CCF-STTG1 cell line, the results indicate that treatment with PAPANONOate decreased cell proliferation $(<50 \%)$ and intracellular trafficking of ceramide, assessed by BODIPY-C5Cer, while these events were not observed in the T98G cell line. Proteomic results suggest that CCF-STTG1 cells are characterized by an increased expression of proteins involved in NO-associated ER stress (i.e. protein disulfide-isomerase A3, calreticulin, $78 \mathrm{kDa}$ glucose-regulated protein), which could compromise ceramide delivery from ER to Golgi, leading to ceramide accumulation in ER and partial growth arrest. Conversely, T98G cell lines, resistant to NO exposure, are characterized by increased levels of cytosolic antioxidant proteins (i.e. glutathione-S-transferase $\mathrm{P}$, peroxiredoxin 1), which might buffer intracellular NO. By providing differential ceramide distribution after NO exposure and differential protein expression of two high grade glioma cell lines, this study highlights specific proteins as possible markers for tumor aggressiveness. This study demonstrates that, in two different high grade glioma cell lines, NO exposure results in a different ceramide distribution and protein expression. Furthermore, this study highlights specific proteins as possible markers for tumor aggressiveness.
\end{abstract}

\section{Introduction}

Anaplastic astrocytomas and glioblastoma multiforme (GBM) are high grade brain tumors originating from astrocytes; ${ }^{1}$ the latter (WHO grade IV) is the most aggressive, being highly invasive and vascularized, with a median survival lower than one year in the $95 \%$ of $_{\text {cases. }}^{2}$ Due to its aggressiveness, chemoresistance and adverse prognosis, the discovery of new therapeutic agents remains a crucial point. Several efforts have

\footnotetext{
${ }^{a}$ Department of Biomedical Sciences for Health, University of Milan, Via Fratelli Cervi 93, Segrate, MI, Italy. E-mail: cecilia.gelfi@unimi.it; Fax: +390221717558

${ }^{b}$ Department of Medical Biotechnology and Translational Medicine, University of Milan, via fratelli Cervi, 93, Segrate, Milan, Italy

${ }^{c}$ Institute of Molecular Bioimaging and Physiology (IBFM), CNR, Cefalù, PA, Italy

${ }^{d}$ IRCCS Policlinico San Donato, Milan, Italy

$\dagger$ Electronic supplementary information (ESI) available. See DOI: 10.1039/ c4mb00725e
}

been made to elucidate the GBM molecular gene signatures; ${ }^{3}$ the results demonstrate that frequent gene mutations, located in many genes, including p53, isocitrate dehydrogenase (IDH), phosphatidylinositol 3-kinase (PI3K) phosphatase and tensin homologue (PTEN), as well as an unmethylated O6-methylguanine methyltransferase (MGMT) promoter, contribute to radio- and chemo-resistance and correlate with poor survival. Furthermore, the infiltrative growth of GBM causes tumor recurrence at the margin of the surgical resection area. ${ }^{3}$

Currently, GBM therapy involves the administration of the alkylating agent temozolomide (TMZ) in conjugation with radiotherapy ${ }^{4}$ or treatment with Carmustine, 1,3-bis(2-chloroethyl)-1nitrosourea (BCNU) in situ, after resection. ${ }^{5}$ Evidence indicates that in gliomas, as in extraglial tumors, the sphingolipid metabolite ceramide (Cer) plays an important role as a tumor suppressor being involved in the action of different chemotherapeutic drugs, ${ }^{6,7}$ nevertheless the ceramide-mediated cell death escape ${ }^{7,8}$ remains a critical point. 
To overcome chemoresistance, new agents in combination with chemotherapy have been considered, among these, nitric oxide (NO) has emerged as a potent chemosensitizer for glioma treatments..$^{9-11} \mathrm{NO}$ is a water-soluble highly reactive free radical, with a major role as a vascular relaxing agent, an inhibitor of platelet aggregation and a neurotransmitter. It can act as an antiinflammatory, a pro-inflammatory or a cytotoxic agent, inducing cell death through apoptosis or necrosis. NO concentration plays a fundamental role in its activity, by a cGMP dependent (at low concentration, i.e. $\mathrm{nM}$ ) or independent (at high concentration, i.e. $1 \mu \mathrm{M})$ mechanism. A previous study demonstrated that treatments with rapid NO releasing donors PAPANONOate $((Z)$ [ $N$-(3-ammoniopropyl)- $N$-(npropyl) amino]diazen-1-ium-1,2-diolate) inhibited rat $\mathrm{C6}$ glioma cell line growth in a dose dependentmanner (via a cGMP-independent mechanism). This inhibition is accompanied by a rapid and significant increase of ceramide in endoplasmic reticulum (ER), presumably, caused by a reduction of ceramide trafficking from ER to Golgi, blunting its conversion into complex sphingolipids. ${ }^{12,13}$ It is also known that the PI3K/Akt (phosphatidylinositol-4,5-bisphosphate 3-kinase/protein kinase B) pathway regulates ceramide metabolism through a positive control of the vesicular ER to Golgi transport. ${ }^{14}$ Other studies, in ovarian cancer cell, demonstrated that NO donors induced a decrement of Akt phosphorylation causing cell death, suggesting that NO donors have the potential to act as anti-tumoral agents, inhibiting cancer cell signaling and reducing cell viability. ${ }^{15}$ In addition, NO may exert an apoptotic effect by increasing ceramide levels through the regulation of metabolic pathways involved in ceramide synthesis or removal. ${ }^{16,17}$ Based on previous results on the rat C6 glioma cell line, the aim of this study was to assess the effect of PAPANONOate considering cell proliferation, ceramide trafficking and proteomic signature of CCF-STTG1 and T98G glioma cell lines. Both cell lines were derived from human gliomas with different oncogene mutations. ${ }^{3}$ CCF-STTG1, as the C6 glioma cell line, has the PI3K/Akt pathway constitutively activated, the former for mutation in PTEN gene $^{9,18}$ and the latter for the lack of PTEN expression, ${ }^{19}$ while the T98G cell line is mutated in p53 (oncosuppressor). ${ }^{20}$

The differential proteomic analysis was based on two dimensional difference in gel electrophoresis (2D-DIGE), MALDI mass spectrometry, coupled with bioinformatics and immunoblotting, to provide quantitative data characteristic of these two cell lines.

Results indicate that T98G compared to the CCF-STTG1 cell line was more resistant to NO and was characterized by a specific proteomic profile. The exposure of the CCF-STTG1 cell line with a nitric oxide donor, PAPANONOate, resulted in a partial inhibition of cell proliferation, which was associated with a rapid and significant accumulation of ceramide at ER levels. Indeed, T98G cells revealed an intracellular microenvironment able to scavenge ROS and a mild UPR (unfolded protein response) activation able to rescue tumor cells from NO exposure. Validation of proteomic results by immunoblotting confirmed the characteristic profile of the T98G cell line and the ability of these cells to survive to NO donors probably by modulating specific proteins. On the other hand, the proteomic profile, validated by immunoblotting analysis, indicates a greater attitude to ER stress response for CCF-STTG1 compared to T98G.

\section{Results}

2.1. Characterization of CCF-STTG1 and T98G cell lines: cell proliferation and Akt signaling

CCF-STTG1, T98G, cell lines were characterized by different proliferating capacity as shown in Fig. 1. CCF-STTG1 showed a duplication rate of 48 hours whereas T98G duplicate in 24 hours. Based on T98G and CCF-STTG1 PTEN states, Akt activation was assessed (Fig. 2). Akt, a downstream effector of PI3K, is hyperactivated when PTEN is mutated or lost. ${ }^{19}$ PTEN acts as a negative control of the PI3K/Akt signaling pathway through its phosphatase activity. The activation of Akt, expressed by the pAkt/Akt ratio, plays an anti-apoptotic role by inducing the overexpression of the antiapoptotic molecule B-cell-lymphoma 2 (Bcl-2). ${ }^{21}$ In CCF-STTG1 cells, Akt was hyperphosphorylated as indicated by the higher pAkt/Akt ratio and $\mathrm{Bcl} 2$ was increased; conversely, in T98G, the pAkt/Akt ratio was very low and $\mathrm{Bcl} 2$ expression was undetectable.

\subsection{Effects of nitric oxide on CCF-STTG1 and T98G cell line proliferation}

The effect of NO exogenously delivered to CCF-STTG1 and T98G, on cell proliferation, was assessed by cell treatment with different concentrations of NO-releasing molecules PAPANONOate, SNAP or SPNO. In CCF-STTG, exposure to NO-releasing molecules caused a $50 \%$ decrease of cell proliferation (Fig. 3A). On the other hand, the same exposure did not alter cell proliferation of T98G cells even when PAPANONOate concentration was drastically increased (up to $1 \mathrm{mM}$ ) (Fig. 3B).

\subsection{Effects of nitric oxide on ceramide distribution in CCF-STTG1 and T98G cell lines}

The effects of NO release on intracellular ceramide trafficking were qualitatively assessed following the redistribution of BODIPY-C5Cer, a fluorescent analogue of ceramide, able to mimic the dynamic of the natural molecule. The distribution of

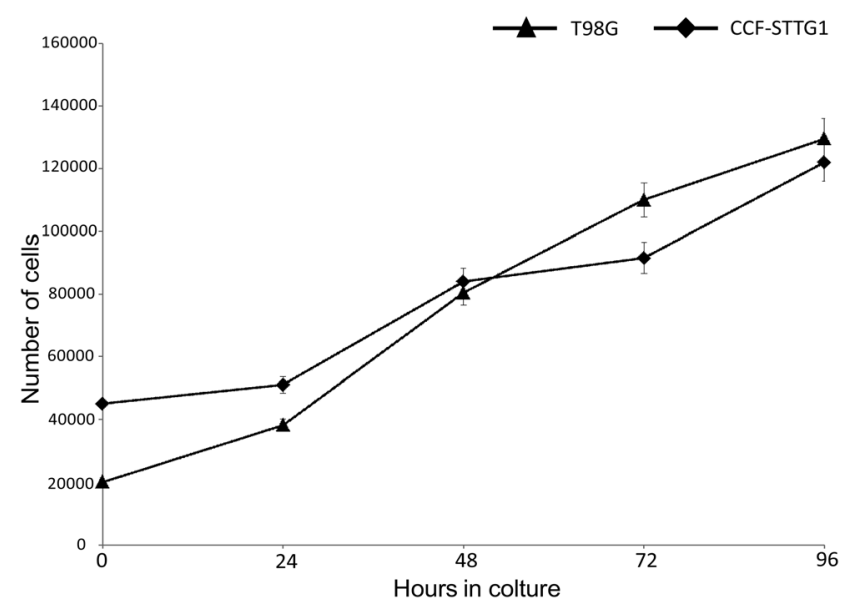

Fig. 1 CCF-STTG1 and T98G glioma cells proliferation. CCF-STTG1, T98G cells were plated and grown in DMEM supplemented with 10\% FCS for 24, 48, 72 and 96 hours. Then, at the indicated times the cells were washed, detached and counted with trypan blue. 

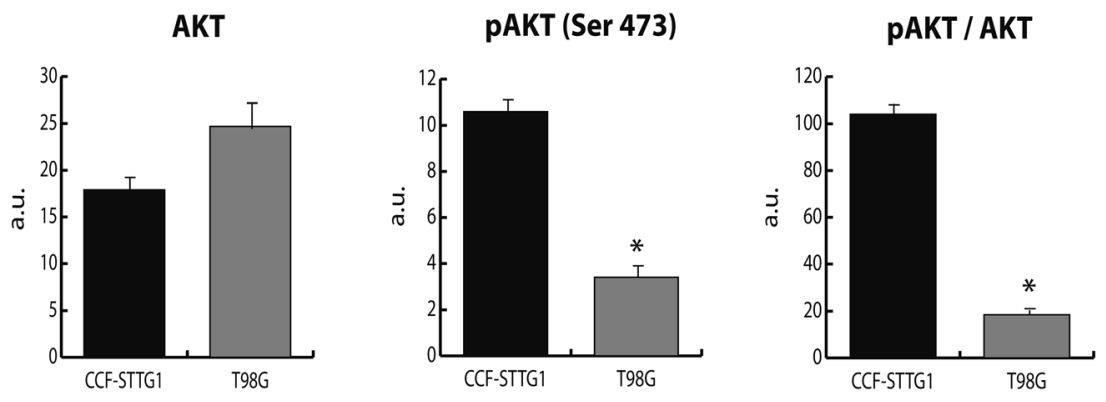

Akt

phospho Akt
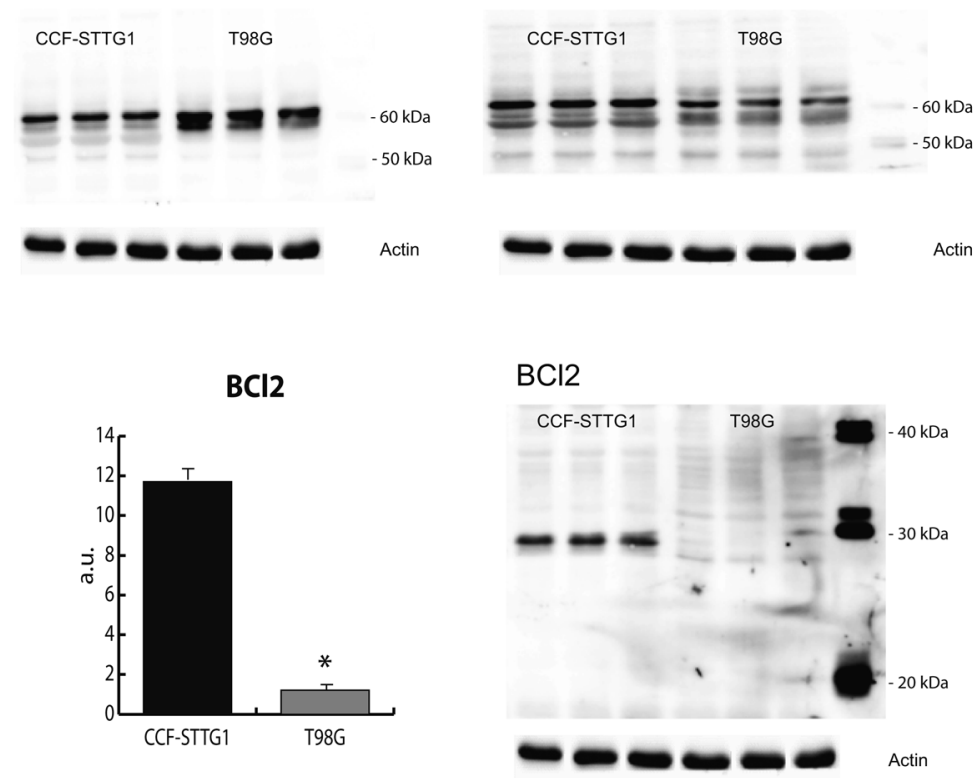

Fig. 2 Assessment of Akt signaling by immunoblotting of T98G and CCF-STTG1 cell line extracts: histograms represent the mean volumes, standard deviations were calculated for the specific band of each experimental group. Data with asterisks indicate statistically significant differences between two cell lines with a $p$ value $<0.05$. Densitometric measurements were normalized against actin. Ratio of pAkt/Akt represents the amount of Akt activated protein.

ceramide in CCF-STTG1 and T98G cells after NO delivery was determined by fluorescence. Cells were treated with BODIPYC5Cer (Fig. 4), and chased in the presence or absence of $0.4 \mathrm{mM}$ PAPANONOate. In CCF-STTG1 untreated cells, fluorescence was accumulated in the perinuclear region, indicative of the Golgi apparatus, (Fig. 4), whereas, as previously observed, ${ }^{13}$ in NO-treated cells fluorescence was spread throughout the cell with reduced accumulation in the Golgi region (Fig. 4). By contrast, in T98G NO-treated cells, BODIPY-C5Cer distribution was similar to control with a prevailing accumulation of fluorescence in the Golgi region (Fig. 4).

\subsection{Proteomic analysis}

The CCF-STTG1 cell line showed a partial decrease of proliferation and ceramide accumulation in ER in response to nitric oxide releasing agent PAPANONOate, whereas the T98G cell line was resistant to NO administration.

The different protein repertoire of CCF-STTG1 and T98G glioma cells was investigated, at the molecular level, by $2 \mathrm{D}$ DIGE and protein identification was obtained by MALDI mass spectrometry. The proteins differentially expressed are listed in
Table S1 (ESI $\dagger$ ). From 2D-DIGE analysis, about 2500 spots per gel were detected, 723 spots were included in the base set for statistical analysis.

The characteristics of these two cell lines, in cell proliferation and ceramide distribution, were confirmed by proteomic results which indicate that among identified spots, 56 were differentially expressed between the two cell lines ( $p$-value $<$ 0.01 ) and only 35 spots with a fold change over 1.5 were considered and grouped according to their functional categories (see Table S1, ESI $\dagger$ ). For each spot, identification UniProtKB accession (AC) numbers, protein name, gene name, theoretical $M_{\mathrm{r}}(\mathrm{kDa}) / \mathrm{pI}$, function and statistical analysis (fold change) are indicated in Table S1 (ESI $\dagger$ ). Fig. S1 (ESI $\dagger$ ) shows a representative map of CCF-STTG1 in panel A and T98G in panel B, the spots differentially changed $(p<0.01$ and fold change $>1.5)$ are indicated by numbers. The proteins, differentially expressed, were grouped according to their functional category: chaperone and stress response proteins, cytoskeletal proteins, intracellular signal proteins, protein degradation, metabolic proteins and other proteins. Mass spectrometry data of identified proteins differentially expressed are listed in Table S2 (ESI $\dagger$ ). 


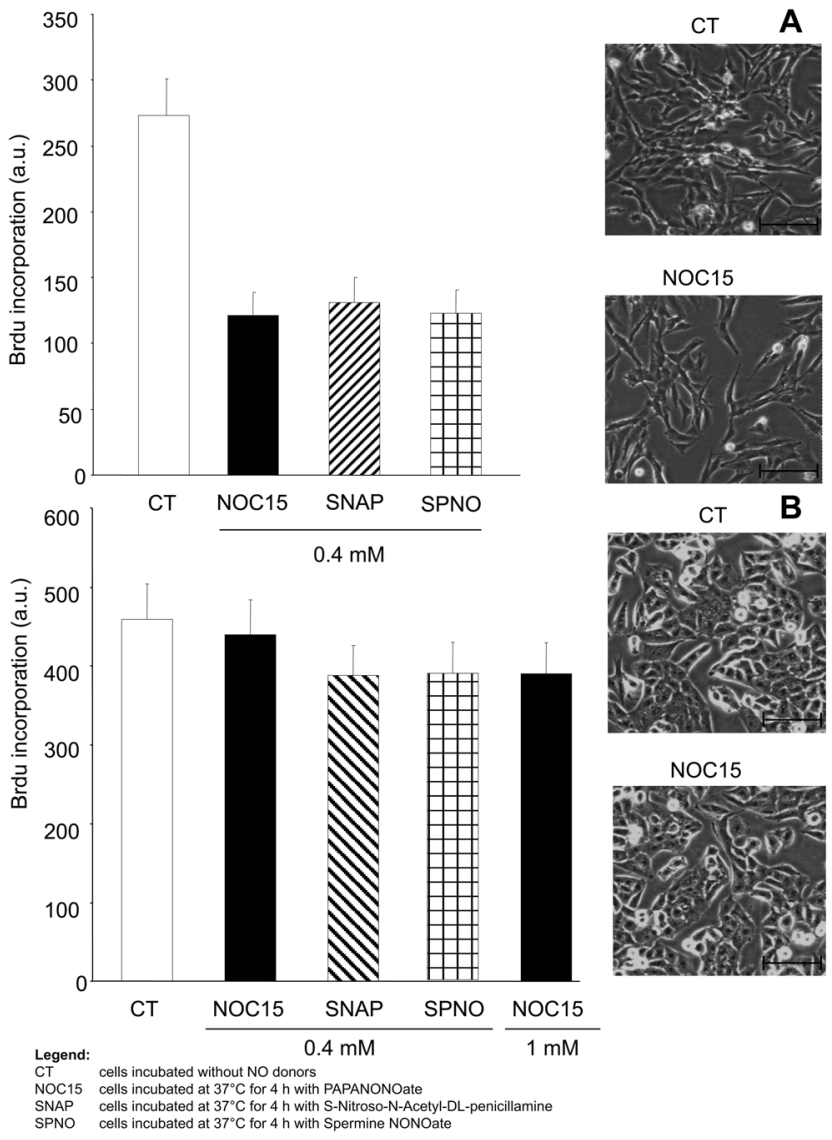

Fig. 3 Effect of NO on CCF-STTG1 and T98G glioma cell proliferation. CCF-STTG1 (panel A), T98G (panel B) cells were grown in DMEM supplemented with $10 \%$ FCS and incubated with different concentrations of NO donors for 4 hours. A chemiluminescence immunoassay for the quantification of cell proliferation based on the measurement of BrdU incorporation during DNA synthesis was used according to manufacturer's instructions (Roche). The incorporated BrdU was detected using a microplate reader. All values are expressed as a mean \pm S.D. of at least three individual experiments. Representative images of CCF-STTG1 and T98G morphology after treatment with or without PAPANONOate. Images were analyzed on a contrast phase microscope and digital images were acquired (magnification, 10x; scale bar, $100 \mu \mathrm{m})$. CT: cells incubated without NO donors; NOC15: cells incubated at $37{ }^{\circ} \mathrm{C}$ for $4 \mathrm{~h}$ with PAPANONOate; SNAP: cells incubated at $37{ }^{\circ} \mathrm{C}$ for $4 \mathrm{~h}$ with $\mathrm{S}$-nitroso-N-acetyl-DL-penicillamine; SPNO: cells incubated at $37{ }^{\circ} \mathrm{C}$ for $4 \mathrm{~h}$ with Spermine NONOate.

2.4.1. Chaperone and stress response proteins (Fig. 5). Comparing T98G vs. CCF-STTG1 cell lines, the HSPs (heat shock proteins) and cytoplasmic stress response proteins HSPA4 (heat shock $70 \mathrm{kDa}$ protein 4), HSP90AB1 (heat shock protein HSP 90-beta), GSTP1 (glutathione $S$-transferase P), PRDX1 (peroxiredoxin 1) were more abundant. Conversely, HSP27 (heat shock protein beta-1) was less abundant and mitochondrial HSPA9 (mortalin) was more abundant in T98G compared to CCF-STTG1. In mitochondria, the interaction of these proteins promotes the control of apoptosis by binding survivin and p53. ${ }^{22}$ Conversely, proteins localized in endoplasmic reticulum, as PDIA3 (protein disulfide isomerase A3), $\mathrm{P} 4 \mathrm{HB}$ (protein disulfide isomerase), CALR (calreticulin), GRP78 (78 kDa glucose-regulated protein) and TRA1 (heat shock protein gp96 precursor), were less abundant in T98G cells compared to CCFSTTG1. CALR, PDI, GRP78 and TRA1 are involved in the unfolding protein response (UPR). ${ }^{23,24}$

2.4.2. Cytoskeletal proteins and intracellular signal proteins (Fig. 6). The majority of the cytoskeletal proteins, such as: F-actin capping protein beta subunit (CAPZB), Vinculin (VLC), and two isoforms of Vimentin, were less abundant in T98G compared to the CCF-STTG1 cell line. Conversely, moesin (MSN) and the Microtubule-associated protein RP/EB family member 1 (MAPRE1) were more abundant in the T98G cell line.

Among proteins involved in intracellular signaling, Rho GDP-dissociation inhibitor 1 (ARHGDIA) and Rab GDP dissociation inhibitor beta (GDI2) were more abundant in CCF-STTG1 than in T98G.

2.4.3. Protein degradation (Fig. 7). Comparing the two cell lines, two subunits of the immunoproteasome activator proteasome activator complex subunit 1 (PSME1), proteasome activator complex subunit 2 (PSME2) and cathepsin D (CTSD), a lysosomal aspartic protease, were less abundant in T98G. Importantly, ubiquitin carboxy-terminal hydrolase L1 (UCHL1) was more abundant in T98G compared to CCF-STTG1 with a fold change of 14.86 .

The global expression of metabolic proteins was similar in the two cell lines, with only a few exceptions like the L-lactate dehydrogenase $\mathrm{B}$ chain and transketolase that were more abundant in the T98G cell line compared to CCF-STTG1.

Galectin 1 (LGALS1) was less abundant in T98G compared to the CCF-STTG1 cell line. Other proteins differentially expressed in T98G vs. CCF-STTG1 are listed in Table S1 (ESI†).

\subsection{GRP78 expression level and localization (Fig. 8)}

The ubiquitously expressed molecular chaperone GRP78 (glucoseregulated protein) is generally localized to the ER (endoplasmic reticulum) and it is specifically induced in cells under UPR. Immunoblotting showed that levels of GRP78 in CCF-STTG1 are twofold of those observed in T98G. Moreover, the GRP78 expression level increased in CCF-STTG1 cells treated with PAPANONOate, but was not modified in T98G cells treated with NO donors (Fig. 8A). Images obtained using fluorescence microscopy demonstrated that in CCF-STTG1 cells under normal growth conditions GRP78 was concentrated in the perinuclear region representative of the ER/Golgi (Fig. 8B) in agreement with previous data. ${ }^{25}$ However, treatments with PAPANONOate decreased its ER targeting, resulting in GRP78 redirection possibly to a cytoplasmic vesicular pattern and mitochondria (Fig. 8B). Conversely T98G cells PAPANONOate did not show modification of their GRP78 level (Fig. 8A) and localization (data not shown).

\section{Discussion}

CCF-STTG1 and T98G cell lines are multidrug-resistant ${ }^{26}$ and NO donors were used to induce growth inhibition and chemosensitization. ${ }^{10}$ In the present study exogenous NO, delivered through PAPANONOate treatment, induced a decrease in cell proliferation $(<50 \%)$ and intracellular trafficking of ceramide 

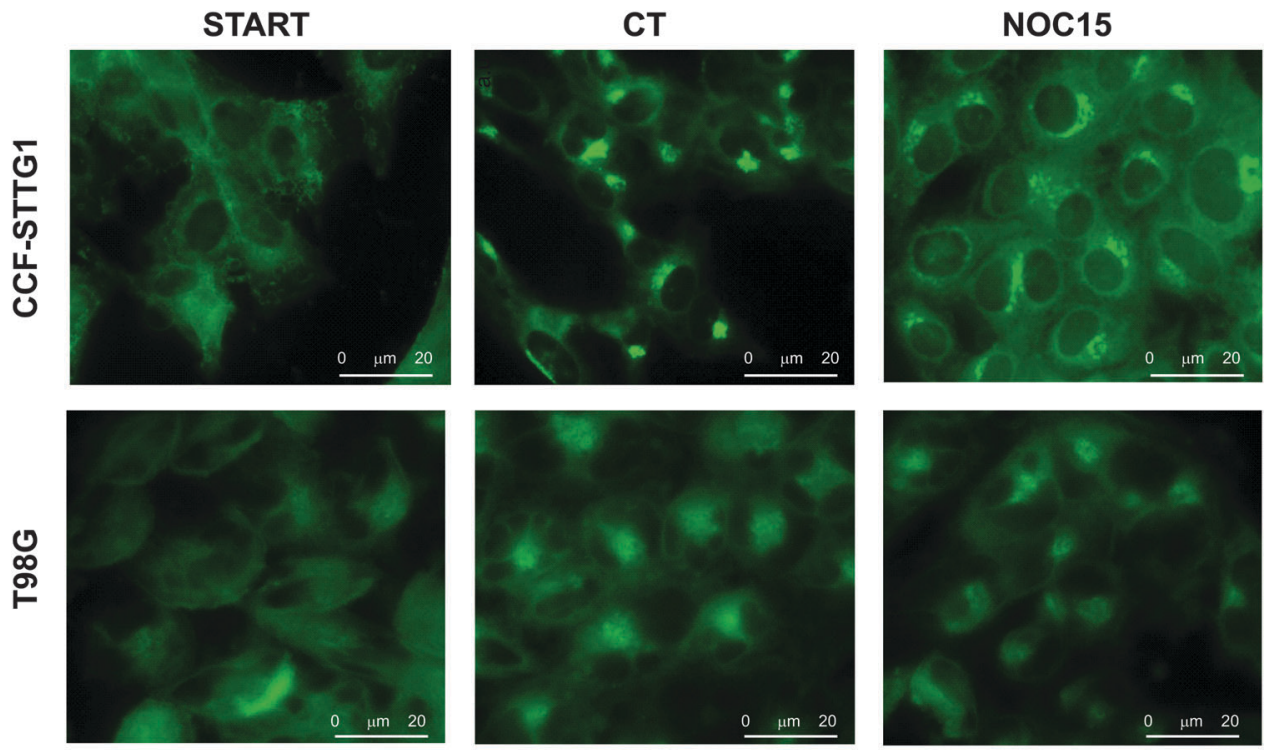

\begin{tabular}{|ll|}
\hline LEGEND \\
START: & cells incubated with BODIPY-C5 Cer/BSA $1: 1(\mathrm{~m} / \mathrm{m})$ in DMEM for $30^{\prime}$ at $4^{\circ} \mathrm{C}$ \\
CT: & cells incubated at $37^{\circ} \mathrm{C}$ for $1 \mathrm{~h}$ without NO donors \\
NOC15: & cells incubated at $37^{\circ} \mathrm{C}$ for $1 \mathrm{~h}$ with PAPANONOate \\
\hline
\end{tabular}

Fig. 4 Effect of NO on intracellular distribution of BODIPY-C ${ }_{5}$ Cer. CCF-STTG1 and T98G cells grown on a glass coverslip were incubated with 5 mM BODIPY $-C_{5}$ Cer/BSA complex $1: 1(\mathrm{~m} / \mathrm{m})$ in DMEM for 30 min at $4{ }^{\circ} \mathrm{C}$ (START). After labeling, cells were incubated at $37{ }^{\circ} \mathrm{C}$ for $1 \mathrm{~h}$ without $(\mathrm{CT})$ and with $400 \mu \mathrm{M}$ PAPANONOate (NOC15). Representative fluorescence microscopy images are shown. All images were processed and printed using the same conditions. Scale bar, $20 \mu \mathrm{m}$.

in CCF-STTG1 but not in the T98G cell line. Results provided by both the Akt pathway and ceramide trafficking analyses suggested a similar profile between human CCF-STTG1 and rat C6 glioma cell lines; ${ }^{13,14}$ whereas the T98G cell line showed a different profile being Akt-harboring and p53 mutated. In addition, Bcl2, a downstream effector of Akt, playing a role in the promotion of tumor malignancy, was not expressed in the T98G cell line, suggesting that these cells may adopt alternative mechanisms to growth and proliferate.

Proteomic analysis, by 2D-DIGE and mass spectrometry, highlighted differences between the two cell lines, which could contribute to explain their different responses to NO exposure. Three main protein functional classes were differently regulated in T98G compared to CCF-STTG1: chaperones and stress response proteins located in the cytosol and endoplasmic reticulum, cytoskeletal proteins and proteins involved in degradation.

T98G was characterized by higher expression levels of stress response proteins, chaperones, redox cytosolic enzymes (i.e. HSPA4, HSP90AB1, GSTP1 and PRDX1) and a mitochondrial protein (HSPA9) and by lower expression levels of ER localized proteins (i.e. PDIA3, P4HB, CALR, GRP78 and TRA1) and of cytoskeleton associated proteins (i.e. HSP27).

Physiologically, molecular chaperones (like HSP90 and HSP70) are known to control protein folding, turnover, differentiation and survival. In particular, it is known that their expression is increased under cellular stress, promoting, in tumors, malignant transformation and maintenance of cancer homeostasis even in a hostile environment, as in the presence of genetic lesions. ${ }^{27}$ In many tumors, the increment of molecular chaperones provides a protection from stress-induced apoptotic signals. ${ }^{28}$ In addition, increased GSTP1 and PRDXs have been associated with an increment of cancer incidence and relapse. ${ }^{22}$ PRDXs are increased in many tumors such as lung, breast cancer, malignant mesothelioma and grade III and IV glioblastomas, ${ }^{27}$ promoting cell proliferation and enhancing resistance to $\mathrm{H}_{2} \mathrm{O}_{2}$ induced apoptosis, radiation or chemotherapeutic treatments. ${ }^{29}$ Due to the different role of chaperone and stress response proteins in tumors compared to normal tissues their increment stimulated tumor aggressiveness ${ }^{27,28}$ leading to hypothesize that the overexpression of cytosolic stress response and redox protein generates an intracellular environment, in the T98G cell line, able to counteract ROS damage and NO activity. Both cell lines expressed ER chaperones involved in UPR response, in particular GRP78, CALR, PDI3, P4HB and TRA1.

Furthermore, P4HB and PDIA3 were differently altered in the two GBM cell lines, being increased in CCF-STTG1 and decreased in T98G. By transplantation of different human glioblastoma biopsies in xenografts, animals developed two different tumor phenotypes: the high infiltrating low angiogenic phenotype characterized by high expression of PDI and the low infiltrating high angiogenic phenotype with low levels of PDI, both phenotypes are representative of the different aspects of human brain tumor biology, in situ..$^{30,31}$

To define the exact role of these molecules further analysis will be required since the effects of PDI in supporting tumor survival and progression could be cell- and cancer-type dependent. The UPR response, in fact, occurs when miss-folded proteins accumulate in ER, generating ER stress, which leads to an increment 

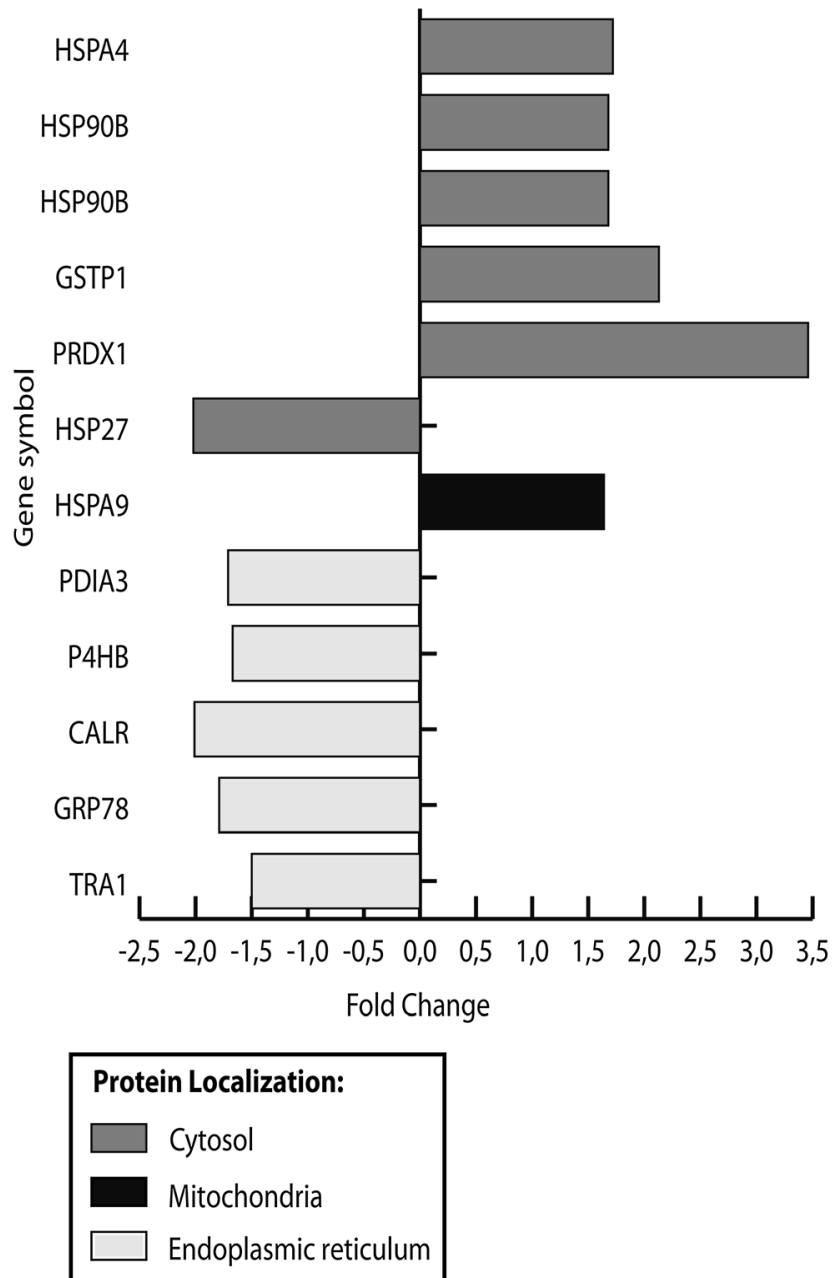

Fig. 5 Histograms of differentially expressed metabolic proteins between T98G and CCF-STTG1 belonging to the chaperones/stress response proteins class. Fold change bars are in gray for cytosolic proteins, black for mitochondrial proteins and light gray for protein localized in ER, respectively. HSPA4 (heat shock $70 \mathrm{kDa}$ protein 4), HSP90B (heat shock protein HSP 90-beta), GSTP1 (glutathione S-transferase P), PRDX1 (peroxiredoxin 1), HSP27 (heat shock protein beta-1), HSPA9 (mortalin), PDIA3 (protein disulfide-isomerase A3), P4HB (protein disulfide isomerase), CALR (calreticulin), GRP78 (78 kDa glucose-regulated protein), TRA1 (heat shock protein gp96 precursor).

of ER chaperone proteins, accompanied by a temporary blockage of protein translation and by the activation of the ERAD (endoplasmic reticulum associated degradation) complex. ${ }^{23}$ In T98G, the levels of proteins controlling the UPR response were lower compared to CCF-STTG1. Elevated protein synthesis, in cancer cell, requires an increase of ER folding, thus an UPR increase in cancer cell (i.e. up regulation of ER chaperones) could provide an advantage for cell proliferation inducing chemoresistance. $^{24}$ Nevertheless, the prolonged and severe activation of UPR leads to cell death by apoptosis. These results could be supportive of a mild activation of UPR that could be an adaptive response, as suggested by T98G cell line behavior. ${ }^{32}$ Indeed, in CCF-STTG1 cells, NO treatment increases ER stress, ${ }^{33}$ promoting ER functional impairment, which, in turn, compromises,
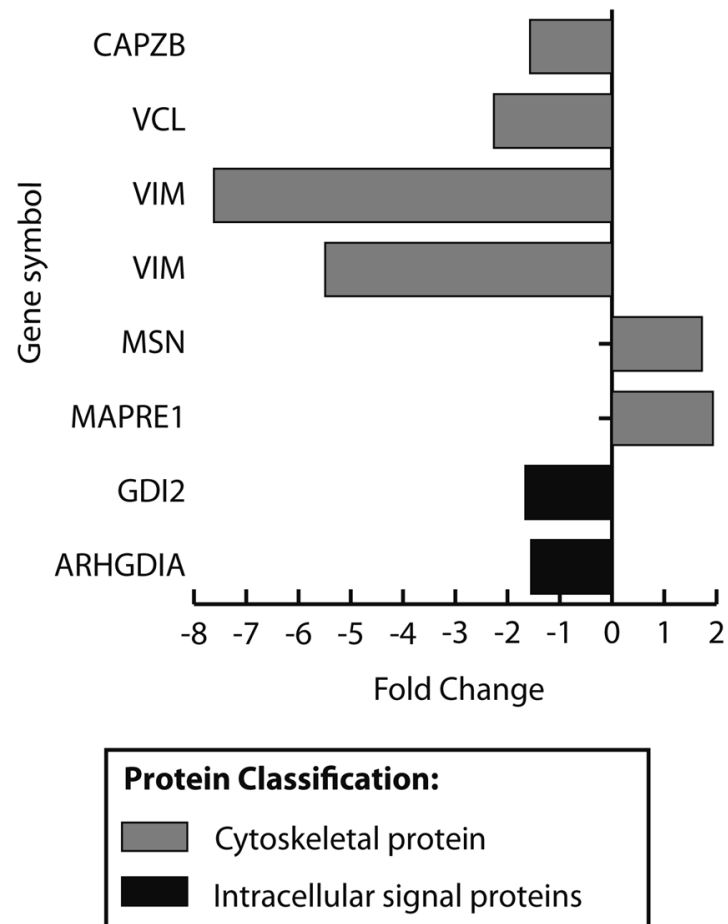

Fig. 6 Histograms of differentially expressed metabolic proteins between T98G and CCF-STTG1, belonging to cytoskeletal and intracellular signal protein functional class. Fold changes bars are indicated in gray for cytoskeletal proteins and black for intracellular signal proteins, respectively. F-actin capping protein beta subunit (CAPZB), vinculin (VCL), vimentin (VIM), moesin (MSN), microtubule-associated protein RP/EB family member 1 (MAPRE1), Rab GDP dissociation inhibitor beta (GDI2) and Rho GDP-dissociation inhibitor 1 (ARHGDIA).

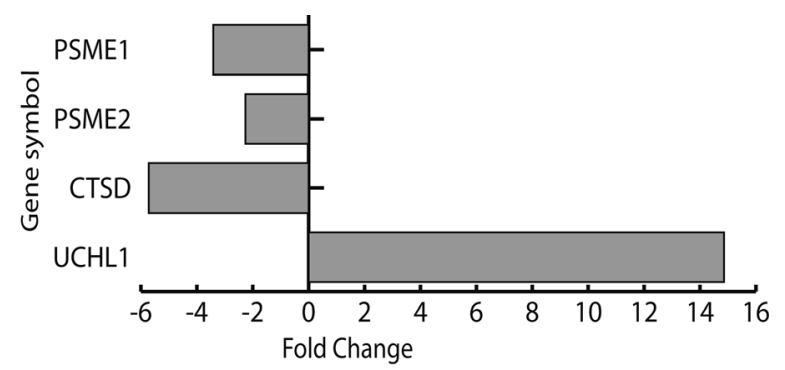

Fig. 7 Histograms of differentially expressed metabolic proteins between T98G and CCF-STTG1 belonging to protein degradation. Bars indicate the fold change. Proteasome activator complex subunit 1 (PSME1), proteasome activator complex subunit 2 (PSME2), cathepsin D (CTSD) and Ubiquitin carboxyl-terminal hydrolase isozyme L1 (UCHL1).

at least partially, the delivery of ceramide from ER to Golgi, with a consequent ceramide accumulation in ER and growth arrest.

The dysregulation of proteins involved in cytoskeletal structure remodeling (i.e. vinculin, moesin and vimentin) and of regulatory proteins (i.e. HSP27, MAPRE1 and ARHGDIA) can be associated with the cytoskeletal reorganization, prominent in T98G compared to the CCF-STTG1 cell line. Vimentin, a marker of EMT (epithelial-mesenchymal transition or transformation) overexpressed in cancer cells, ${ }^{34}$ was significantly less abundant in T98G compared to CCF-STTG1 cell lines. Moreover moesin 
A
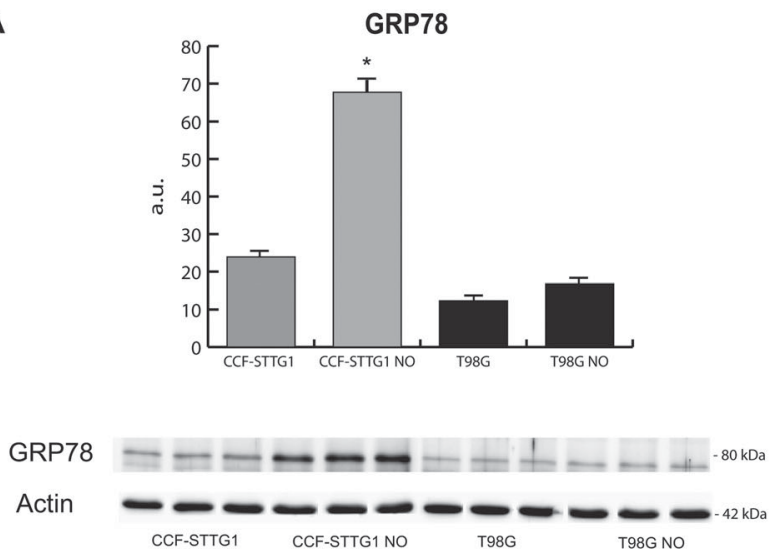

B

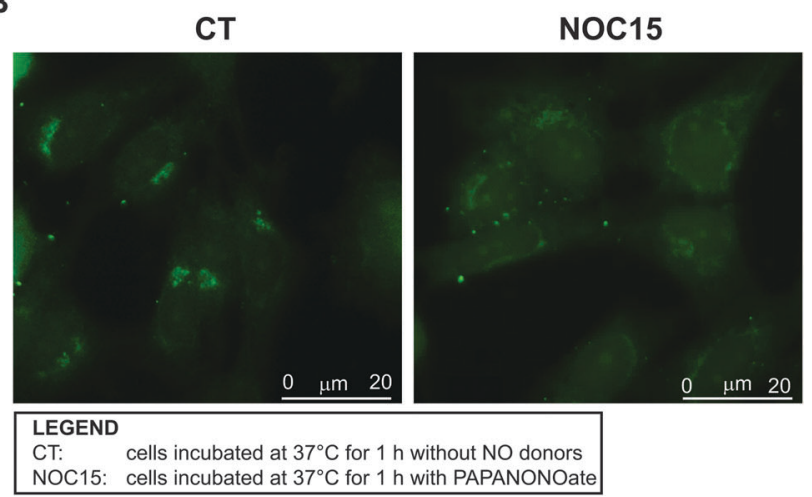

Fig. 8 Immunoblotting of GRP78 on CCF-STTG1 and T98G cells before and after PAPANONOate treatment. Histograms represent the mean volumes and the standard deviations calculated for the specific band of each experimental group. Data with asterisks indicate significant differences between two cell lines with a $p$ value $<0.05$ (panel A). The effect of NO on intracellular GRP78 localization. CCF-STTG1 and T98G cells grown on a glass coverslip were incubated at $37{ }^{\circ} \mathrm{C}$ for $4 \mathrm{~h}$ with and without $400 \mu \mathrm{M}$ PAPANONOate. Cells were stained with the anti-GRP78 antibody followed by the FITC-conjugated secondary antibody. Representative fluorescence microscopy images are shown. All images were processed and printed under the same conditions. Scale bar, $20 \mu \mathrm{m}$ (panel B).

(MSN), involved in actin cytoskeleton control and cell migration, known to promote GBM proliferation and invasiveness, ${ }^{35}$ and the Microtubule-associated protein RP/EB family member 1 (MAPRE1), a master regulator of the microtubule, ${ }^{36}$ were more abundant in the T98G cell line. It can be assumed that, in T98G cells, this decrement may be due to a higher protein catabolism instead of a decreased synthesis. This hypothesis is supported by the low levels of heat shock proteins (HSP27 was less abundant in T98G than CCF-STTG1) and by the cell turnover rate of $\mathrm{T} 98 \mathrm{G},{ }^{26}$ which induces a persistent remodeling of structural proteins.

The Rho family-Rho GDI system plays a crucial role in actin cytoskeletal control and its down-regulation leads to Rho GTPase activation signaling. ${ }^{37}$ The insensitivity to NO of T98G cells may be due to a decrement of GDI2, a negative regulator of the monomeric G protein Rab GTPases which inhibits the exchange of GDP to GTP, stabilizing Rab proteins associated with GDP. Rab proteins are involved in the vesicular trafficking within cells, particularly Rab1 and Rab2 are implicated in endoplasmic reticulum to Golgi apparatus transport. ${ }^{38}$ The negative regulator GDI2 may contribute to blunt the sensitivity of T98G to nitric oxide on ceramide distribution by affecting the ceramide vesicular transport between intracellular compartments.

Finally, also levels of PSME1, PSME2, cathepsin D, acting as regulators of proteasomal degradation and promoters of oxidative stress adaptation, ${ }^{39,40}$ and Galectin 1 have been found altered.

Galectin 1 has been found dysregulated in many tumors including astrocytoma, melanoma, prostate, colon and ovary carcinomas and is often correlated with tumor aggressiveness and metastatization. ${ }^{37}$ In T98G cells, the above proteins were less abundant compared to CCF-STTG1, suggesting that due to the partial effect of NO in CCF-STTG1 (50\% decrement of cell proliferation), treatments able to modulate these proteins/ pathways should be associated with NO donors to improve treatment response.

Furthermore, the differential expression between T98G and CCF-STTG1 of ubiquitin carboxy-terminal hydrolase L1 (UCHL1), more abundant in T98G (fold change +14.86), has to be considered. The overexpression of UCHL1 leads to a down regulation of E-cadherin and consequently anoikis resistance, independent growth, anchoring and spreading. UCHL1 regulates key processes in tumor progression and, particularly, the signaling of $\beta$-catenin/TCF stabilizing the latter by de-ubiquitination. The $\beta$-catenin/TCF mediates the expression of genes like c-myc, cyclin $\mathrm{D}, \mathrm{c}$-jun, and survivin and others that are part of the oncogenic pathway. Growing evidence suggests that UCHL1 is overexpressed in many tumors and is associated with a poor prognosis. ${ }^{38}$ Recent results demonstrate that the selective inhibition of UCHL1 by small molecules and siRNA causes a decrease in tumor proliferation and migration and increases the sensitivity of chemotherapeutic agents inducing apoptosis. ${ }^{41}$

Overall these results suggest that nitric oxide treatment is a potential anti-oncogenic agent that could overcome the resistance to conventional therapeutic agents in some cancer cells. The NO releasing donor, PAPANONOate, causes ceramide accumulation in CCF-STTG1 cell lines. This accumulation can induce a switching of the Akt hyperactivated signal, ${ }^{14,42}$ causing growth inhibition. From proteomic data, the UPR stress response was more pronounced in CCF-STTG1 than T98G, and this feature could interfere with ER and Golgi trafficking, in particular under nitric oxide stress.

\section{Materials and methods}

Materials: Dulbecco's modified Eagle's medium (DMEM), L-glutamine, sodium pyruvate solution, penicillin/streptomycin, bovine serum albumin fraction $\mathrm{V}$ (BSA), fatty acid freeBSA, phosphate buffer saline (PBS) were from Sigma-Aldrich (St. Louis, MO, USA). Fetal calf serum (FCS) was from Euroclone (Pero, Milano, Italy). (Z)-[N-(3-Ammoniopropyl)- $N$-(npropyl)amino]diazen-1-ium-1,2-diolate (PAPANONOate), $S$-nitroso- $N$-acetyl-DLpenicillamine (SNAP) and (Z)-1-[N-[3-aminopropyl]- $N$-[4-(3aminopropylammonio)butyl]-amino]diazen-1-ium-1,2-diolate 
(SPNO) were from Alexis-Italia (Firenze, Italy). $N$-(4,4,-Difluoro5-,7-dimethyl-bora-3a,4a-diaza-s-indacene-3-pentanoyl) sphingosine BODIPY-C ${ }_{5}$ Cer was from Molecular Probes Europe (Leiden, The Netherlands). Bromodeoxyuridine (BrdU) was from Roche Italia (Milan, Italy). Primary mouse polyclonal goat anti-GRP78 and FITCconjugated rabbit anti-goat secondary antibody were from Santa Cruz Biotechnology (Santa Cruz, CA, USA).

\subsection{Cell cultures}

Two human glioblastoma cell lines were used: the cell line CCFSTTG1, p53 wild type and PTEN mutated, and the T98G cell line p53 mutated and PTEN wild type obtained from American Type Culture Collection (Rockville, MD). The two cell lines were grown at $37{ }^{\circ} \mathrm{C}$ in DMEM supplemented with 10\% FCS, $1 \mathrm{mM}$ sodium pyruvate, $2 \mathrm{mM}$ L-glutamine, $100 \mathrm{U} \mathrm{ml}^{-1}$ penicillin, $100 \mu \mathrm{g} \mathrm{ml}^{-1}$ streptomycin and $0.25 \mu \mathrm{g} \mathrm{ml}^{-1}$ amphotericin B in a fully humidified incubator containing $5 \% \mathrm{CO}_{2}$ and $95 \%$ air. For proteomic analysis 2D-DIGE, CCF-STTG1 and T98G cells were plated $\left(6 \times 10^{4}\right.$ cells per $\mathrm{cm}^{2}$ and $1.5 \times 10^{4}$ cells per $\mathrm{cm}^{2}$ respectively) and maintained in DMEM plus $10 \%$ FCS for $72 \mathrm{~h}$.

\subsection{Proliferation assay}

CCF-STTG1 and T98G cells were plated $\left(6 \times 10^{4}\right.$ cells per $\mathrm{cm}^{2}$, $1.5 \times 10^{4}$ cells per $\mathrm{cm}^{2}$ respectively) and maintained in DMEM plus $10 \%$ FCS for $24 \mathrm{~h}$. To assess proliferation the cells were grown in DMEM plus 10\% FCS for 24, 48, 72 and 96 hours then, at the indicated times, the cells were washed twice with PBS, detached by Trypsin/EDTA $(0.025 / 0.001 \%)$ and counted with trypan blue. To evaluate the effect of NO the cells were then incubated in DMEM plus 10\% FCS for $4 \mathrm{~h}$ with or without the following NO donors: 0.4-1 mM PAPANONOate, 0.4 mM SNAP, $0.4 \mathrm{mM}$ SPNO. Based on C6 glioma cell results, a PAPANONOate concentration of $0.4 \mathrm{mM}$ was selected due to its maximal inhibitory effect on cell proliferation. ${ }^{13} \mathrm{~A}$ chemiluminescence immunoassay for the quantification of cell proliferation based on the measurement of BrdU incorporation during DNA synthesis was used according to the manufacturer's instruction (Roche). The BrdU incorporated was detected using a microplate reader (Wallac Victor2 from PerkinElmer).

\subsection{Analysis of the intracellular distribution of fluorescent ceramides}

CCF-STTG1 and T98G cells plated at $4.5 \times 10^{4}$ cell per $\mathrm{cm}^{2}$ and $1.5 \times 10^{4}$ cell per $\mathrm{cm}^{2}$, respectively, were grown on a glass coverslip. Then cells were incubated with $5 \mu \mathrm{M}$ BODIPY-C5Cer (as a $1: 1$ complex with fatty acid free BSA) in DMEM at $4{ }^{\circ} \mathrm{C}$ for $30 \mathrm{~min}$ and analyzed as previously described. ${ }^{13}$ Briefly, after washing with DMEM plus $10 \%$ FCS and $0.34 \mathrm{mg} \mathrm{ml}^{-1}$ fatty acidfree BSA, cells were incubated $1 \mathrm{~h}$ at $37{ }^{\circ} \mathrm{C}$ in DMEM plus $10 \%$ FCS with or without PAPANONOate. Cells were then washed and fixed with $0.5 \%$ glutaraldehyde solution in PBS for $10 \mathrm{~min}$ at $4{ }^{\circ} \mathrm{C}$. The specimens were immediately observed and analyzed using a fluorescence microscope (Olympus BX-50) equipped with a fast high resolution charge-coupled device camera (Colorview 12) and dedicated software for image analysis (analysis from Soft Imaging System $\mathrm{GmbH}$ ).

\subsection{Proteomic analysis}

4.4.1. Two dimensional difference in gel electrophoresis (2D-DIGE). Protein labeling, 2D separation and analysis were performed as previously described. ${ }^{43}$ The pellet of each sample was suspended in lysis buffer, assayed and labelled with the CyDye fluorophores. A common internal standard for the two cell lines was created. The internal standard was labeled with Cy3, while samples were labeled with Cy5 and analyzed in triplicate. The first dimension was performed on a IPGstrip310 nonlinear (NL) immobilized $\mathrm{pH}$ gradient, $24 \mathrm{~cm}$ long, each strip was loaded with $40 \mu \mathrm{g}$ of internal standard and $40 \mu \mathrm{g}$ of the sample. Gel images were processed by 2D DeCyder software for the quantitative analysis. Statistically significant differences of 2D-DIGE data were computed by Student's $t$-test $(p<0.01)$. To minimize false-positives, data were filtered by the following criteria: false discovery rate (FDR) and 1.5 fold difference in abundance (fold change). FDR correction was applied as a multiple test for the correction method to keep the overall error rate as low as possible. ${ }^{44}$ The fold change, provided by DeCyder software analysis, indicates the value derived from the normalized spot volume standardized against the intra-gel standard.

4.4.2. Protein identification by MALDI-ToF mass spectrometry. Proteins were identified by peptide mass fingerprinting (PMF) utilizing a matrix-assisted laser desorption/ionization (MALDI) time-of-flight (TOF) mass spectrometer (Bruker Daltonics), as previously described. ${ }^{43}$ In particular, search was carried out by correlation of uninterpreted spectra with Mammalia entries in the NCBInr 20070216 database (4626804 sequences; 1596079197 residues).

4.4.3. Bioinformatic data analysis of molecular pathways. The MetaCore software (GeneGo, Inc., V6.3 build 25177), using two different bioinformatics analyses ("Enrichment Ontologies" and "Analyze Network"), was used for the interpretation of differential protein expression from CCF-STTG1 and T98G cell lines and for searching protein-protein interaction networks.

\subsection{Immunoblottings}

Protein extracts $(50 \mu \mathrm{g})$ from CCF-STTG1 and T98G cell lines were loaded in triplicate and resolved on $12 \%$ polyacrylamide gels, according to protein molecular weight. Membranes were incubated with antibodies diluted as follows: polyclonal anti Akt (cell signaling \#9272) 1 : 1000, polyclonal anti Phospho-Akt (Ser473 cell signaling \#9271) $1: 1000$, anti BCl2 (cell signaling \#2876) 1 : 1000. For each gel MagicMark ${ }^{\mathrm{TM}}$ XP Western Protein Standard (Life Technologies LC5602) was loaded. To confirm 2D-DIGE data, some selected differential expressed proteins were validated by immunoblotting (Fig. S2, ESI $\dagger$ ). After washing, membranes were incubated with anti-rabbit (GE Healthcare) or anti-goat (Santa Cruz Biotechnology) secondary antibodies conjugated with horseradish peroxidase. Signals were visualized by chemiluminescence using the ECL Plus detection kit. Images were acquired using a LAS 4000 mini (GE Healthcare) and were analysed using Image Quant TL 
(molecular dynamics). The data were normalized against actin (Santa Cruz sc-7210 diluted 1:1000) and analyzed through a Student's $t$-test $(p<0.05)$.

\subsection{Analysis of intracellular localization of GRP78 by fluorescence microscopy}

CCF-STTG1 and T98G cells plated at $6 \times 10^{4}$ cell per $\mathrm{cm}^{2}$ and $1.5 \times 10^{4}$ cell per $\mathrm{cm}^{2}$ respectively were grown on a glass coverslip for 24 hours. Then the cells were treated with $0.4 \mathrm{mM}$ PAPANONOate for $4 \mathrm{~h}$ and fixed with $0.5 \%$ glutaraldehyde solution in PBS for $10 \mathrm{~min}$ at $4{ }^{\circ} \mathrm{C}$. The cells were then permeabilized with $0.2 \%$ Triton $\mathrm{X}-100$ for $30 \mathrm{~min}$ at room temperature, then blocked for $1 \mathrm{~h}$ with $0.2 \%$ Triton X-100, 5\% BSA, 1\% FCS in PBS. After washing the cells were incubated 1 hour with the primary antibody anti-GRP78 and stained with the secondary FITC-conjugated antibody. Coverslips were mounted on glass slides and the specimens were analyzed using a fluorescence microscope (Olympus BX-50) equipped with a fast high resolution CCD camera (Colorview 12) and image analytical software (Soft Imaging System GmbH, Olympus, Munster, Germany).

\section{Conclusions}

Nitric oxide is emerging as a potential anti-oncogenic agent to overcome chemo- and radio-resistance in cancer cells; although its actions depend on the cell microenvironment. The treatment with the NO donor, PAPANONOate, is able to partially reduce the proliferation of CCF-STTG1, suggesting that NO alone is not sufficient to inhibit the tumor cell growth. In CCF-STTG1, an overexpression of proteins located in the endoplasmic reticulum and involved in the unfolded protein response was observed, making this cell line sensible to NO. By contrast, in the T98G cell line, a complex of cytosolic antioxidant and stress response proteins provides resistance to NO activity. This study, by providing differential ceramide distribution and differential protein expression, following NO exposure, identifies specific proteins as possible tumor aggressiveness markers of two human high grade glioma cell lines.

\section{Disclosure of potential conflicts of interest}

No potential conflicts of interest were disclosed.

\section{Acknowledgements}

This work was supported by The Ministry of University and Scientific Research (Grant: FIRB RBN No. 7BMCT to Prof. Cecilia Gelfi) and the PhD School in molecular and translational medicine of University of Milan and SysbioNet, a MIUR grant for the Italian Roadmap of European Strategy Forum on Research Infrastructures (ESFRI).

\section{References}

1 P. C. Burger and B. W. Schithauer, Armed Forces Institute of Pathology, Washington, D.C., 1994.

2 D. N. Louis, H. Ohgaki, O. D. Wiestler, W. K. Cavenee, P. C. Burger, A. Jouvet, B. W. Scheithauer and P. Kleihues, Acta Neuropathol., 2007, 114, 97-109.

3 C. G. A. R. Network, Nature, 2008, 455, 1061-1068.

4 J. E. Chang, H. I. Robins and M. P. Mehta, Clin. Adv. Hematol. Oncol., 2007, 5, 54-64.

5 G. Noel, R. Schott, S. Froelich, M. P. Gaub, P. Boyer, D. Fischer-Lokou, P. Dufour, P. Kehrli and D. Maitrot, Int. J. Radiat. Oncol., Biol., Phys., 2012, 82, 749-755.

6 G. Velasco, A. Carracedo, C. Blazquez, M. Lorente, T. Aguado, A. Haro, C. Sanchez, I. Galve-Roperh and M. Guzman, Mol. Neurobiol., 2007, 36, 60-67.

7 P. Giussani, R. Bassi, V. Anelli, L. Brioschi, F. De Zen, E. Riccitelli, M. Caroli, R. Campanella, S. M. Gaini, P. Viani and L. Riboni, Cancer Invest., 2012, 30, 27-37.

8 C. A. Dumitru, M. Weller and E. Gulbins, J. Cell. Physiol., 2009, 221, 688-695.

9 S. Safdar, C. A. Payne, N. H. Tu and L. J. Taite, Biotechnol. Bioeng., 2013, 110, 1211-1220.

10 A. Weyerbrock, B. Baumer and A. Papazoglou, J. Neurosurg., 2009, 110, 128-136.

11 S. Huerta, S. Chilka and B. Bonavida, Int. J. Oncol., 2008, 33, 909-927.

12 R. N. Kolesnick, F. M. Goni and A. Alonso, J. Cell. Physiol., 2000, 184, 285-300.

13 P. Viani, P. Giussani, L. Brioschi, R. Bassi, V. Anelli, G. Tettamanti and L. Riboni, J. Biol. Chem., 2003, 278, 9592-9601.

14 P. Giussani, L. Brioschi, R. Bassi, L. Riboni and P. Viani, J. Biol. Chem., 2009, 284, 5088-5096.

15 M. Kielbik, M. Klink, M. Brzezinska, I. Szulc and Z. Sulowska, Nitric Oxide, 2013, 35, 93-109.

16 A. Huwiler, J. Pfeilschifter and H. van den Bosch, J. Biol. Chem., 1999, 274, 7190-7195.

17 Y. Takeda, M. Tashima, A. Takahashi, T. Uchiyama and T. Okazaki, J. Biol. Chem., 1999, 274, 10654-10660.

18 R. Zhang, N. L. Banik and S. K. Ray, Neurochem. Res., 2007, 32, 2194-2202.

19 T. Kubiatowski, T. Jang, M. B. Lachyankar, R. Salmonsen, R. R. Nabi, P. J. Quesenberry, N. S. Litofsky, A. H. Ross and L. D. Recht, J. Neurosurg., 2001, 95, 480-488.

20 M. Alonso, C. Tamasdan, D. C. Miller and E. W. Newcomb, Mol. Cancer Ther., 2003, 2, 139-150.

21 S. Pugazhenthi, A. Nesterova, C. Sable, K. A. Heidenreich, L. M. Boxer, L. E. Heasley and J. E. Reusch, J. Biol. Chem., 2000, 275, 10761-10766.

22 G. Di Pietro, L. A. Magno and F. Rios-Santos, Expert Opin. Drug Metab. Toxicol., 2010, 6, 153-170.

23 N. Naidoo, Ageing Res. Rev., 2009, 8, 150-159.

24 P. Pyrko, A. H. Schonthal, F. M. Hofman, T. C. Chen and A. S. Lee, Cancer Res., 2007, 67, 9809-9816.

25 F. C. Sun, S. Wei, C. W. Li, Y. S. Chang, C. C. Chao and Y. K. Lai, Biochem. J., 2006, 396, 31-39. 
26 V. C. Chumbalkar, C. Subhashini, V. M. Dhople, C. S. Sundaram, M. V. Jagannadham, K. N. Kumar, P. N. Srinivas, R. Mythili, M. K. Rao, M. J. Kulkarni, S. Hegde, A. S. Hegde, C. Samual, V. Santosh, L. Singh and R. Sirdeshmukh, Proteomics, 2005, 5, 1167-1177.

27 D. R. Ciocca and S. K. Calderwood, Cell Stress Chaperones, 2005, 10, 86-103.

28 L. Whitesell and S. L. Lindquist, Nat. Rev. Cancer, 2005, 5, 761-772.

29 L. Kim and M. Glantz, Curr. Treat. Options Oncol., 2006, 7, 467-478.

30 D. Goplen, J. Wang, P. O. Enger, B. B. Tysnes, A. J. Terzis, O. D. Laerum and R. Bjerkvig, Cancer Res., 2006, 66, 9895-9902.

31 S. Xu, S. Sankar and N. Neamati, Drug Discovery Today, 2014, 19, 222-240.

32 H. R. Jin, J. Zhao, Z. Zhang, Y. Liao, C. Z. Wang, W. H. Huang, S. P. Li, T. C. He, C. S. Yuan and W. Du, Cell Death Dis., 2012, 3, e376.

33 W. Xu, I. G. Charles and S. Moncada, Cell Res., 2005, 15, 63-65. 34 S. Valastyan and R. A. Weinberg, Cell, 2011, 147, 275-292.

35 K. Ovaska, M. Laakso, S. Haapa-Paananen, R. Louhimo, P. Chen, V. Aittomaki, E. Valo, J. Nunez-Fontarnau,
V. Rantanen, S. Karinen, K. Nousiainen, A. M. LahesmaaKorpinen, M. Miettinen, L. Saarinen, P. Kohonen, J. Wu, J. Westermarck and S. Hautaniemi, Genome Med., 2010, 2, 65.

36 S. Etienne-Manneville, Curr. Opin. Cell Biol., 2010, 22, 104-111.

37 A. Danguy, I. Camby and R. Kiss, Biochim. Biophys. Acta, 2002, 1572, 285-293.

38 T. Frisan, G. Coppotelli, R. Dryselius and M. G. Masucci, FASEB J., 2012, 26, 5060-5070.

39 Y. S. Hah, H. S. Noh, J. H. Ha, J. S. Ahn, J. R. Hahm, H. Y. Cho and D. R. Kim, Cancer Lett., 2012, 323, 208-214.

40 A. M. Pickering and K. J. Davies, Arch. Biochem. Biophys., 2012, 523, 181-190.

41 A. Bheda, J. Shackelford and J. S. Pagano, PLoS One, 2009, 4, e6764.

42 M. J. Zinda, C. J. Vlahos and M. T. Lai, Biochem. Biophys. Res. Commun., 2001, 280, 1107-1115.

43 A. Vigano, M. Vasso, A. Caretti, V. Bravata, L. Terraneo, C. Fania, D. Capitanio, M. Samaja and C. Gelfi, Proteomics, 2011, 11, 4202-4217.

44 D. Drai, Y. Benjamini and I. Golani, J. Neurosci. Methods, 2000, 96, 119-131. 